\title{
1. Aussenpolitik der Schweiz
}

\section{Christoph Stamm}

\section{(2) OpenEdition \\ Journals}

Electronic version

URL: http://journals.openedition.org/sjep/250

DOI: $10.4000 /$ sjep. 250

ISSN: 1663-9677

\section{Publisher}

Institut de hautes études internationales et du développement

\section{Printed version}

Date of publication: 1 avril 2003

Number of pages: $3-11$

ISBN: 2-88247-050-3

ISSN: $1660-5926$

\section{Electronic reference}

Christoph Stamm, «1. Aussenpolitik der Schweiz », Schweizerisches Jahrbuch für Entwicklungspolitik [Online], 22-1 | 2003, Online erschienen am: 04 Mai 2010, abgerufen am 08 September 2020. URL : http://journals.openedition.org/sjep/250 ; DOI : https://doi.org/10.4000/sjep.250 


\section{AUSSENPOLITIK DER SCHWEIZ*}

Z WEI WICHTIGE EREIGNISSE prägten die Schweizer Aussenpolitik im Jahr 2002. Zum einen ist die Schweiz, nach einem positiven Volksentscheid, der Organisation der Vereinten Nationen im September 2002 als Vollmitglied beigetreten. Zum anderen wurden mit dem In-Kraft-Treten der bilateralen Verträge im Juni 2002 die Beziehungen zur Europäischen Union (EU) auf eine neue Grundlage gestellt. Mit dem gleichzeitigen Beginn neuer Verhandlungen (Bilaterale II) wird der bilaterale Weg zwischen der Schweiz und der EU fortgeführt.

Damit geht ein aus aussenpolitischer Sicht bewegtes Jahrzehnt zu Ende, das 1992 mit der Ablehnung des EWR und dem Beitritt zur Weltbankgruppe und zum Internationalen Währungsfonds begonnen hatte. Hinweise auf eine Neuausrichtung der Aussenpolitik finden sich auch in der Sicherheits- und Friedenspolitik. Erstmals standen bei einer Friedensmission (Kosovo-Friedenstruppe KFOR) bewaffnete Schweizer Soldaten im Einsatz, und die zivile Friedensförderung wird mit einem Bundesgesetz auf eine solide Basis gestellt.

\subsection{DIE SCHWEIZ UND DIE UNO}

\section{$\square$ Abstimmung zum UNO-Beitritt}

Die Stimmberechtigten befürworteten am 3. März 2002 die eidgenössische Volksinitiative für den Beitritt der Schweiz zur Organisation der Vereinten Nationen (UNO) mit einem Ja-Anteil von 54,6 Prozent. Bei einer Stimmbeteiligung von 58 Prozent stimmten 12 Stände für und 11 Stände gegen den Beitritt ${ }^{1}$. Dies bedeutet die kleinstmögliche Mehrheit für die Erreichung des Ständemehrs.

Im Vergleich zur Referendumsabstimmung gegen den UNO-Beitritt von 1986 eine Mitgliedschaft wurde damals vom Volk und von allen Ständen massiv abgelehnt - hat sich der Ja-Anteil bei der aktuellen Abstimmung um über 20 Prozent erhöht. Die Diskrepanz zwischen den beiden Abstimmungsresultaten ist ein Hinweis auf den grossen Stimmungsumschwung, der in den letzten 16 Jahren und vor allem seit dem Ende des Kalten Krieges erfolgt ist.

Mit der Ermächtigung von Volk und Ständen konnte der Bundesrat am 20. Juni 2002 die Organisation der Vereinten Nationen um die Aufnahme der Schweizerischen Eidgenossenschaft ersuchen².

메 JSDW 2002, S. 207-209.

* Von Christoph Stamm, Politologe.

1 Bund, Vorlage Nr. 485, Eidgenössische Volksinitiative für den Beitritt der Schweiz zur UNO, <www. admin.ch>.

2 Bundesrat, Gesuch zum Beitritt der Schweiz zur UNO, mit Neutralitätserklärung, Juni 2002, <www. uno.admin.ch>. 


\section{$\square$ Die UNO-Politik der Schweiz}

Vor dem offiziellen Beitritt zur UNO formulierte der Bundesrat im Mai 2002 seine UNO-Politik und legte die Schwerpunkte für die 57. Session der UNOGeneralversammlung fest ${ }^{3}$.

Die Schweiz besitzt nach dem Beitritt in der UNO-Generalversammlung das passive und das aktive Wahlrecht. Sie kann in den Wirtschafts- und Sozialrat (ECOSOC), dessen Kommissionen sowie in den Sicherheitsrat gewählt werden. Der Bundesrat möchte eine Kandidaturplanung aufstellen, welche sowohl den Einsitz der Schweiz in internationalen Gremien als auch Personenkandidaturen erfasst. Im Vordergrund steht dabei die Wahl in den ECOSOC und in die Menschenrechtskommission. Dazu muss sich die Schweiz aber um die Aufnahme in teilweise langfristige Rotationsmechanismen der UNO-Mitglieder bemühen.

Schwergewichte können ausserdem durch allgemeine Interventionen in der Generaldebatte der Vollversammlung beziehungsweise in den Unterausschüssen, durch Einbringung und Mitunterzeichnung von Resolutionen und durch Mitwirkung an den informellen Konsultationen zur Vorbereitung der Resolutionen und Beschlüsse gesetzt werden ${ }^{4}$.

Die schweizerische Haltung in der UNO orientiert sich an den Vorgaben des Aussenpolitischen Berichts 2000, und namentlich an den dort formulierten aussenpolitischen Prioritäten: Wahrung und Förderung von Sicherheit und Frieden; Abbau sozialer Gegensätze; Förderung der Wohlfahrt; Förderung von Menschenrechten, Demokratie und Rechtsstaat; Schutz der natürlichen Lebensgrundlagen. Auch die Festigung der Bedeutung des UNO-Sitzes in Genf bildet einen Schwerpunkt der Schweiz ${ }^{5}$.

Rund 20 Schweizer Nichtregierungsorganisationen haben eine Plattform gebildet, um sich aktiv am UNO-Prozess beteiligen zu können ${ }^{6}$. Mit einem ersten Dokument stellten sie ihre Forderungen im August 2002 an den Bundesrat. Die NRO legten den Finger auf die in ihren Augen ungenügende Informationspolitik des Bundes und auf die sich zwangsläufig ergebenden Kohärenzprobleme zwischen den verschiedenen Politikfeldern. In diesem Zusammenhang stehen für die Plattform der Nichtregierungsorganisationen die Grund- und Menschenrechte, soziale Standards und die Ziele der Nachhaltigkeit im Vordergrund. Die NRO machten darauf aufmerksam, dass die Schweiz weder die Alpenkonvention noch das Kyoto-Protokoll zur Klimakonvention ratifiziert habe.

Im Hinblick auf die 57. Session der Generalversammlung waren den NRO zwei Anliegen besonders wichtig: Erstens soll der Internationale Strafgerichtshof so schnell wie möglich operativ werden und zweitens soll die Schweiz das in der UN-Charta verankerte Gewaltmonopol der UNO anerkennen. Dies

3 Pressemitteilung des Bundes, Der Bundesrat definiert seine UNO-Politik und legt die Schwerpunkte für die 57. Session der UNO-Generalversammlung fest, Bern: 29. Mai 2002.

4 Resolutionen sind Empfehlungen und entfalten keine unmittelbare rechtliche Bindung; Beschlüsse regeln rein prozedurale Fragen.

5 EDA, 57. UNO-Generalversammlung 2002, Erste Session mit Schweizer Mitgliedschaft, Bern 2002, Seite 11 .

6 Plattform der Schweizerischen NGO zuhanden des Bundesrates anlässlich der ersten UNO-Generalversammlung mit Schweizer Vollmitgliedschaft, Gesellschaft Schweiz-Vereinte Nationen, Bern : 26. August 2002. 
bedeute insbesondere, dass die Schweiz friedenserhaltende Operationen nur dann befürworten und sich allenfalls im Rahmen ihrer Möglichkeiten beteiligen kann, wenn diese von der UNO oder der OSZE beschlossen werden.

\section{$\square$ Der UNO-Beitritt der Schweiz}

Die Schweiz wurde am 10. September 2002 anlässlich des Eröffnungstages der jährlichen UNO-Generalversammlung aufgrund einer Empfehlung des Sicherheitsrates vom 24. Juli 2002 als 190. Vollmitglied in die UNO aufgenommen ${ }^{7}$. Der französische Aussenminister, der die Aufnahmeresolution der Schweiz im Namen der Nachbarländer vorlegte, wies in seiner Rede darauf hin, dass die Schweiz das einzige Land sei, dessen Beitritt zur UNO durch eine Volksabstimmung entschieden worden sei, und dass dies dazu beitragen könne, die demokratische Ausrichtung der UNO zu stärken.

In seiner Erklärung vor der Generalversammlung betonte Bundespräsident Kaspar Villiger den Willen der Schweiz, im Rahmen der UNO in einem konstruktiven und kritischen Geist mitzuarbeiten. Er betonte auch einige zentrale Elemente der schweizerischen politischen Identität und unterstrich schliesslich, dass die Schweiz auch weiterhin ein neutrales Land bleiben werde ${ }^{8}$.

Mit dem UNO-Beitritt der Schweiz und Osttimors (am 25. September 2002) ist die Zahl der Mitgliedsstaaten der UNO auf 191 angestiegen. Alle von der internationalen Gemeinschaft als unabhängig anerkannten Staaten, mit Ausnahme des Vatikans, sind nun Mitglieder der Organisation der Vereinten Nationen.

\section{$\square$ Erste Aktivitäten an der Generalversammlung der UNO}

Am Tag des Schweizer UNO-Beitritts eröffnete die Generalversammlung ihre 57. Session?. Die Generalversammlung ist eines der Hauptorgane der UNO. Sie besitzt eine umfassende Zuständigkeit hinsichtlich der Erörterung aller Fragen und Angelegenheiten, welche in den Rahmen der UNO-Charta fallen. Neben politischen Funktionen nimmt die alljährlich zusammentretende Generalversammlung auch organisatorische Aufgaben wahr ${ }^{10}$. Die ordentliche Jahressession beginnt jeweils am zweiten Septemberdienstag mit einer zweiwöchigen Generaldebatte. Danach werden die Arbeiten in den sechs Ausschüssen oder im Plenum bis ins neue Jahr weitergeführt.

An der Generaldebatte wurde unter anderem über ein vom Generalsekretär vorgelegtes Reformpaket, über die Reform des Sicherheitsrates, den Stellenwert des Multilateralismus und über eine verbesserte Zusammenarbeit der Akteure in der humanitären Hilfe diskutiert. Ausserdem stand die Wahl von fünf neuen nichtständigen Mitgliedern des Sicherheitsrates auf dem Programm ${ }^{11}$. Die Schweiz nahm an den Diskussionen und Wahlen teil. Sie begrüsste die Lancierung des Reformpakets, zu dessen Zielen namentlich die Stärkung der Kapazitäten der UNO beim Schutz und bei der Förderung der Menschenrechte, die

\footnotetext{
Neue Zürcher Zeitung, „,Grosser Tag für die Schweiz in New York“, 11. September 2002.

8 EDA, Rede von Bundespräsident Kaspar Villiger zum UNO-Beitritt der Schweiz an der UNO-Vollversammlung, New York: 10. September 2002.

9 EDA, Die Schweiz in der UNO: Zwischenbilanz - Verlauf der Arbeiten der Generalversammlung, Oktober 2002, <www.uno.admin.ch>.

10 General Assembly, About the General Assembly, <www.un.org/ga/57/about.htm>.

11 Für zwei Jahre gewählt wurden Deutschland, Spanien, Chile, Angola und Pakistan.
} 
Rationalisierung und die Verbesserung der Kommunikation sowie eine Verringerung der Anzahl der Sitzungen und Berichte gehört. Was die Wahlen und Kandidaturen betrifft, so hat sich der UNO-Beitritt der Schweiz bereits ausgewirkt: Professor Walter Kälin wurde als Mitglied des UNO-Komitees für Menschenrechte gewählt; der Diplomat Valentin Zellweger wurde zum Vizepräsidenten des sechsten Ausschusses der Generalversammlung ernannt und schliesslich wurde Botschafter Jean-Marc Boulgaris zum Vizepräsidenten des Exekutivkomitees des Hochkommissariats der Vereinten Nationen für Flüchtlinge ernannt ${ }^{12}$.

In den sechs thematischen Ausschüssen der Generalversammlung, in denen die verschiedensten Resolutionsentwürfe diskutiert und verabschiedet werden, kann die Schweiz zu Sachfragen Stellung nehmen und ihre welt- und aussenpolitischen Anliegen einbringen.

- Erster Ausschuss: Sicherheit und Abrüstung

Die Schweiz will sich für die Eliminierung von Massenvernichtungswaffen und für eine bessere Transparenz auf dem Gebiet der Bewaffnung einsetzen. Sie fördert zudem auf dem Gebiet der menschlichen Sicherheit eine mit Frankreich entwickelte Initiative bezüglich des Auffindens leichter und kleinkalibriger Waffen.

- Zweiter Ausschuss: Entwicklung, Finanzen und Umwelt

Der Bund unterstützt die neue Strategie für die Entwicklung Afrikas (NEPAD) $)^{13}$; er setzt sich für eine bessere Zusammenarbeit zwischen den verschiedenen von Entwicklungsfragen betroffenen Akteuren (Regierungen, internationale Organisationen, Zivilgesellschaft und Privatsektor) ein und wünscht sich auf dem Gebiet des Umweltschutzes eine bessere Umsetzung der bestehenden Abkommen.

- Dritter Ausschuss: soziale, kulturelle und humanitäre Fragen

Die Schweizer Diplomaten messen der Annahme eines Zusatzprotokolls gegen die Folter durch die Generalversammlung besondere Bedeutung zu.

- Vierter Ausschuss : besondere politische Fragen und Entkolonialisierung Dieser Ausschuss befasst sich hauptsächlich mit der Friedenserhaltung (Verbesserung der UNO-Missionen) und mit besonderen politischen Fragen, wie zum Beispiel der Lage im Nahen Osten. Die Schweiz setzt sich für die Einhaltung der Genfer Konvention in den besetzten Gebieten ein.

- Fünfter Ausschuss : Budget- und Personalfragen

Die Schweiz setzt sich für ein Nullwachstum des ordentlichen Budgets ein. Sie befürwortet aber eine Umschichtung der Ressourcen zu Gunsten der Verteidigung der Menschenrechte und des Umweltschutzes. Der für zwei Drittel aller Ausgaben verantwortliche Budgetposten für die friedenserhaltenden Massnahmen soll transparenter werden.

- Sechster Ausschuss: Rechtsfragen

Der Diskussion über den Internationalen Strafgerichtshof sowie der Genfer

12 Pressemitteilung des Bundes, Professor Kälin in den Menschenrechtsausschuss der UNO gewählt, Bern: 9. September 2002.

Pressemitteilung des Bundes, Schweizer Diplomat wird stellvertretender Vorsitzender des UNO-Ausschusses für Völkerrecht, Bern: 24. September 2002.

13 Siehe JSDW 2002, S. 205. 
Konvention und ihren Zusatzprotokollen kommt die besondere Aufmerksamkeit des Bundes zu.

Insgesamt wird die Schweiz in ihrem ersten UNO-Jahr zu rund 300 Resolutionen Stellung nehmen. Dabei sind die Schweizer Diplomaten um Botschafter Jenö Staehelin gefordert, trotz Ad-hoc-Strukturen und begrenzten Ressourcen die verschiedenen Resolutionsentwürfe rasch zu behandeln.

\subsection{BUNDESGESETZZUR ZIVILEN FRIEDENSFÖRDERUNG UND ZUR STÄRKUNG DER MENSCHENRECHTE}

Das Eidgenössische Departement für auswärtige Angelegenheiten (EDA) wurde aufgrund eines Bundesratsbeschlusses vom Juni 2000 beauftragt, einen Entwurf für ein Bundesgesetz über Massnahmen zur zivilen Friedensförderung und zur Stärkung der Menschenrechte auszuarbeiten. Der Bundesrat überwies dem Parlament den Gesetzesentwurf und die entsprechende Botschaft (02.077) am 23. Oktober $2002^{14}$.

Das Gesetz war notwendig geworden, weil der Bund in den fraglichen Bereichen Budgetmittel in Höhe von 40 Millionen Franken ausgibt, ohne dass für diese Aufgaben eine formell-gesetzliche Grundlage besteht. Das Bundesgesetz hat vornehmlich technischen Charakter und umfasst weder inhaltliche noch programmatische Neuausrichtungen ${ }^{15}$. Letztere werden jeweils im Zusammenhang mit den Anträgen für die nun gesetzlich vorgeschriebenen Rahmenkredite festgelegt.

Daher hat der Bundesrat, parallel zum Bundesgesetz, eine Botschaft über einen Rahmenkredit für Massnahmen zur zivilen Konfliktbearbeitung und Menschenrechtsförderung (02.076) an die Eidgenössischen Räte überwiesen. Der Kredit weist ein Verpflichtungsvolumen von 240 Millionen Franken auf. Dem EDA wird damit ermöglicht, seine Aktivitäten in den beiden Politikfeldern im Sinne einer mehrjährigen Ressourcenplanung zu optimieren. Das jährliche Budget soll sukzessive von 44,5 Millionen im Jahr 2003 auf 62,5 Millionen im Jahr 2007 erhöht werden ${ }^{16}$. In der Botschaft über den Rahmenkredit werden nach einer Umfeldanalyse und einem Rückblick auf die bisherigen Bemühungen die künftigen Ziele, Grundsätze, Aktionsfelder sowie die thematischen Schwerpunkte festgelegt.

Jahrbuch 2003, Nr. 1, Kap. 11, „Friedens- und Sicherheitspolitik“.

Zum Bereich Menschenrechtsförderung gehören die beiden Aktionsfelder Diplomatische Initiativen und Menschenrechtsdialog. Die zivile Friedensförderung beinhaltet die Aktionsfelder Gute Dienste und Vermittlung, Programme der

14 Das neue Gesetz soll auf den ersten Januar 2004 in Kraft treten.

Pressemitteilung des Bundes, Bundesgesetz und Rahmenkredit für Massnahmen zur zivilen Friedensförderung, Bern: 23. Oktober 2002.

15 Schwerpunkte und Instrumente für die Friedensförderung siehe: EDA, Konzept für friedensfördernde Massnahmen Legislaturperiode 2000-2003, Dezember 1999, und EDA, Gewalt vorbeugen Frieden mitgestalten Demokratie stärken (Die Friedesnpolitik der Politischen Direktion EDA), Juli 2001; für die Menschenrechts-Aussenpolitik siehe: Bundesrat, Bericht über die Menschenrechtspolitik der Schweiz, Februar 2000.

16 Neue Zürcher Zeitung, „Mehr Mittel für zivile Friedensförderung“, 24. Oktober 2002. 
zivilen Konfliktbearbeitung, Expertenpool für zivile Friedensförderung, Thematische diplomatische Initiativen und Partnerschaften (zum Beispiel Entwicklung friedenspolitischer Strategien). Die Bereiche Verfassungsfragen/Dezentralisierung/Machtteilung, Medien und Gewaltkonflikte und Menschliche Sicherheit gelten als Schwerpunktthemen.

Der Bundesrat legte, ebenfalls am 23. Oktober 2002, einen Bericht über die Möglichkeiten und Grenzen von freiwilligen Auslandeinsätzen im Rahmen der zivilen Friedensförderung vor $^{17}$. Der Bericht misst dem im Jahr 2000 gegründeten schweizerischen Expertenpool für zivile Friedensförderung (SEF) besonderen Stellenwert zu. Die Erfahrungen mit der Entsendung der „Friedensexperten“ wird als positiv beurteilt. Der Bundesrat ist aber bemüht, die Vorbereitung, Begleitung und Auswertung solcher Auslandeinsätze zu verbessern.

\subsection{BILDUNG EINER EIDGENÖSSISCHEN MENSCHENRECHTSKOMMISSION}

Bereits 1993 hatte die Internationale Menschenrechtskonferenz in ihrem von der Schweiz unterstützten Aktionsplan die Wichtigkeit nationaler Menschenrechtskommissionen betont. Aber erst seit dem Erscheinen eines Berichts des Bundesrates über die Menschenrechtspolitik der Schweiz im Februar 2000 ist die Frage der Schaffung einer Eidgenössischen Menschenrechtskommission von Aktualität. Rund hundert Nichtregierungsorganisationen fordern die Bildung einer unabhängigen Instanz, die für die Respektierung der Menschenrechte in der Schweiz sorgt ${ }^{18}$. Im Dezember 2001 wurde im National- und im Ständerat eine parlamentarische Initiative eingereicht, die die Schaffung einer Menschenrechtskommission verlangt. Die Aussenpolitische Kommission des Ständerates hat die Initiative im September 2002 abgelehnt und stattdessen den Bundesrat mit der Erstellung eines Berichts über die mögliche Schaffung einer Menschenrechtskommission beauftragt ${ }^{19}$. Die Staatspolitische Kommission des Nationalrates dagegen empfahl, einer entsprechenden Initiative zuzustimmen. Der Nationalrat wird sich in der Wintersession 2002 zur Initiative äussern. Da bereits 109 der 200 Parlamentarier die Initiative unterschrieben haben, sollte sich im Nationalrat eine Mehrheit finden ${ }^{20}$.

[DI JSDW 2001, Bericht über die Menschenrechtspolitik der Schweiz, S. 271-273.

Für einen Teil der Menschenrechte (Rassismusbekämpfung, Gleichstellung von Mann und Frau) gibt es bereits Kontrollinstrumente in der Schweiz ${ }^{21}$. Eine Kommission für alle wirtschaftlichen, sozialen, kulturellen, politischen und bürgerlichen Rechte fehlt jedoch. Gemäss einem Bericht der Initianten könnte eine Menschenrechtskommission folgende Aufgaben erfüllen: Beobachtung und

17 Pressemitteilung des Bundes, Bericht des Bundesrates über die Möglichkeiten und Grenzen von freiwilligen Auslandeinsätzen im Rahmen der zivilen Friedensförderung, Bern : 23. Oktober 2002.

18 Arbeitsgemeinschaft Menschenrechtskommission (Hg.), Eidgenössische Kommission für Menschenrechte, Bern: September 2002.

19 Bundesparlament, Postulat 02.3394 der Aussenpolitischen Kommission, Eidgenössische Kommission für Menschenrechte, 9. September 2002.

20 Tagesanzeiger, „Die Menschenrechte im eigenen Land durchsetzen“, 4. Oktober 2002.

${ }^{21}$ Die im Eidgenössischen Departement des Innern angesiedelten Institutionen sind die Eidgenössische Kommission gegen Rassismus, die Fachstelle für Rassismusbekämpfung und das Eidgenössische Büro für die Gleichstellung von Mann und Frau. 
Begleitung der Umsetzung der internationalen Menschenrechtsverträge in der Schweiz; juristische und politische Aufarbeitung von Menschenrechtsverletzungen, Initiierung von Lösungsfindungsprozessen, Beratung des Parlaments und der Regierung in Menschenrechtsfragen, Sensibilisierung der Öffentlichkeit für Menschenrechtsfragen und Beiträge zur Stärkung und Weiterentwicklung der nationalen und internationalen Instrumente des Menschenrechtsschutzes ${ }^{22}$.

Menschenrechtspolitik betrifft sowohl die Innen- als auch die Aussenpolitik. Indem die mögliche Menschenrechtskommission ihre Beobachtungs- und Beratungstätigkeit auch für die Aussenpolitik wahrnimmt, könnte sie zu einer aus menschenrechtlicher Sicht kohärenteren Innen- und Aussenpolitik beitragen.

\subsection{AUSSENPOLITISCHE SÜDOSTEUROPA-STRATEGIE DER SCHWEIZ}

Der Bundesrat hat am 24. April 2002 eine Strategie für Südosteuropa ${ }^{23}$ verabschiedet $^{24}$. Diese (nach der bereits am 11. April 2001 verabschiedeten Strategie für den südlichen und östlichen Mittelmeerraum) zweite Strategie dieses Typs trägt den im Aussenpolitischen Bericht 2000 definierten geografischen Prioritäten der Aussenpolitik Rechnung.

Die Bedeutung der Stabilität der Region für Europa und die Auswirkungen der Kriege und Krisen der letzten zehn Jahre in der Region haben die Schweiz dazu veranlasst, dort substanzielle und vielfältige Mittel einzusetzen. Die Strategie identifiziert die Interessen der Schweiz in der Region, setzt die Ziele ihrer mittelfristigen Politik (3-5 Jahre) fest und erinnert an die Instrumente, die ihr zur Erreichung dieser Ziele zur Verfügung stehen. Die Hauptinteressen der Schweiz in der Region sind folgende: Prävention neuer bewaffneter Konflikte; Entwicklung von Südosteuropa zum vollwertigen politischen und ökonomischen Partner; Prävention von massiven Flüchtlings- und Migrationsströmen; Kampf gegen den politischen Extremismus und das organisierte Verbrechen und die Integration in die euro-atlantischen Strukturen. Hauptanliegen der aussenpolitischen Strategie ist die Verbesserung der Koordination der schweizerischen Aktionen in Südosteuropa ${ }^{25}$. Gemäss der Strategie ist die Schweiz ein wichtiger Partner in der Region. Die Schweiz werde, solange es die Situation erfordere, ihren Teil an der internationalen Zusammenarbeit übernehmen, und sei entschlossen, dieser Priorität die notwendigen Mittel zukommen zu lassen.

22 Arbeitsgemeinschaft Menschenrechtskommission (Hg.), Eidgenössische Kommission für Menschenrechte, Bern: September 2002.

23 Die Region Südosteuropa umfasst folgende Länder: Albanien, Bosnien-Herzegowina, Bulgarien, Kroatien, die Republik Mazedonien, Moldawien, die Bundesrepublik Jugoslawien sowie Rumänien.

24 Bundesrat, Aussenpolitische Südosteuropa-Strategie der Schweiz, Bern: April 2002.

25 Folgende Departemente und Bundesämter sind in der Region aktiv: Departement für auswärtige Angelegenheiten, Direktion für Entwicklung und Zusammenarbeit, Staatssekretariat für Wirtschaft, Bundesamt für Flüchtlinge, Departement für Verteidigung, Bevölkerungsschutz und Sport. Die Koordination obliegt dem EDA. 


\subsection{AUSSENPOLITIK IM WANDEL}

Den aussenpolitischen Veränderungen des letzten Jahrzehnts widmet sich ein Buch mit dem Titel Schweizerische Aussenpolitik Grundlagen und Möglichkei$\operatorname{ten}^{26}$. Die Autoren fragen zum einen nach den Auswirkungen des globalen Wandels auf die Aussenpolitik der Schweiz und beschäftigen sich zum anderen mit den aussenpolitischen Entscheidungsprozessen und dem Einfluss innenpolitischer Faktoren auf die Aussenpolitik. Das Werk stützt sich dabei auf die Arbeiten des Ende 2000 abgeschlossenen Nationalen Forschungsprogramms Grundlagen und Möglichkeiten der schweizerischen Aussenpolitik (NFP 42) ${ }^{27}$.

Gemäss den Autoren hat sich der seit Ende des Kalten Krieges beschleunigte Wandel des internationalen Umfelds in zweifacher Hinsicht auf die Aussenpolitik der Schweiz ausgewirkt: „Einerseits hat der Multilateralismus an Bedeutung gewonnen, andererseits hat die Verschränkung zwischen Innen- und Aussenpolitik zugenommen. Daraus resultieren zusätzliche Zielkonflikte und Herausforderungen an die Gestaltung einer kohärenten Aussenpolitik" ${ }^{28}$. Die neue Ausrichtung der Aussenpolitik (sie betrifft nicht ausschliesslich die Schweiz) ist gekennzeichnet durch eine Zunahme der Anzahl und der Art der Akteure, das verstärkte Aufkommen aussenpolitischer Themen, welche sowohl die globale Gesellschaft als auch das Individuum betreffen, eine grössere Transparenz und eine durch die Inhalte bedingte neue Rechenschaftspflicht. Diese Merkmale sind in der Schweiz durch die aussenpolitischen Mitentscheidungsrechte (Staatsvertragsreferendum) der Bevölkerung akzentuiert. Hinzu kommt, dass die für die Innenpolitik konzipierten direktdemokratischen Rechte (Volksinitiative und Gesetzereferendum) immer häufiger aussenpolitische Themen betreffen.

Gehe die entsprechende Entwicklung weiter, so die Autoren, „wird die Bezeichnung der Aussenpolitik als Weltinnenpolitik nicht mehr nur Gedankenspiel sein, sondern eine neue Realität“'29. und Möglichkeiten, Bern: Verlag NZZ, 2002.

27 Programmleitung Laurent Goetschel, siehe NFP 42: <www.snf.ch/NFP/nfp42/home.htm>.

28 Goetschel, Bernath, Schwarz 2002, S. 222.

29 Goetschel, Bernath, Schwarz 2002, S. 228. 


\section{QUELLEN}

Goetschel Laurent, Aussenpolitik im Wandel, Synthese des NFP 42, Bern: Oktober 2000.

Goetschel Laurent, Bernath Magdalena, Schwarz Daniel, Schweizerische Aussenpolitik Grundlagen und Möglichkeiten, Bern: Verlag NZZ, 2002.

Bund, Vorlage Nr. 485, Eidgenössische Volksinitiative für den Beitritt der Schweiz zur Organisation der Vereinten Nationen, Bern: 2002, <www.admin.ch/ch/d/pore/va>.

Bund, Vorlage Nr. 338, Bundesbeschluss über den Beitritt der Schweiz zur Organisation der Vereinten Nationen, Bern: 1986, <www.admin.ch/ch/d/pore/va>.

Bundesrat, Bericht über die Menschenrechtspolitik der Schweiz, Bern: Februar 2000.

Bundesrat, Aussenpolitische Südosteuropa-Strategie der Schweiz, Bern: April 2002.

Bundesrat, Botschaft über Massnahmen zur zivilen Friedensförderung und Stärkung der Menschenrechte, Bern: Oktober 2002.

EDA, Konzept für friedensfördernde Massnahmen Legislaturperiode 2000-2003, Bern : Dezember 1999.

EDA, Gewalt vorbeugen Frieden mitgestalten Demokratie stärken (Die Friedenspolitik der Politischen Direktion EDA), Bern: Juli 2001.

EDA, 57. UNO-Generalversammlung 2002, Erste Session mit Schweizer Mitgliedschaft, Bern, 2002.

EDA, Massnahmen zur zivilen Friedensförderung und Stärkung der Menschenrechte, Bern: 23. Oktober 2002.

EDA, Die Schweiz und die UNO: Zwischenbilanz - Verlauf der Arbeiten der Generalversammlung, Bern, 2002.

EDA, Schweiz global, Das Magazin des Departements für auswärtige Angelegenheiten, Ausgabe 2/2002 und 4/2002, Bern, 2002.

EDA, Menschenrechte, Bern, 2002.

Arbeitsgemeinschaft Menschenrechtskommission (Hg.), Eidgenössische Kommission für Menschenrechte, Bern: September 2002.

Menschenrechte Schweiz (Hg.), humanrights.ch Nr. 3, Bern : September 2002.

Plattform der Schweizerischen NGO zuhanden des Bundesrates anlässlich der ersten UNO-Generalversammlung mit Schweizer Vollmitgliedschaft, Gesellschaft Schweiz-Vereinte Nationen, Bern: August 2002.

Neue Zürcher Zeitung, 24. Oktober 2002, 11. September 2002.

Tagesanzeiger, 4. Oktober 2002.

\section{INTERNET-ADRESSEN}

EDA - Informationen zur UNO: <www.uno.admin.ch $>$.

Menschenrechte Schweiz MERS : <www.humanrights.ch>.

Organisation der Vereinten Nationen: <www.un.org $>$.

Schweizerische UNO-Mission in New York: <www.eda.admin.ch.newyork_miss/e/home >

Schweizerische UNO-Mission in Genf: <www.eda.admin.ch.geneva_miss/e/home>.

UN, General Assembly, Fifty-Seventh Session : 2002: <www.un.org/ga/57/index.html>.

Vereinte Nationen, Informationszentrum Bonn: <www.uno.de $>$. 